\title{
Spatiotemporal Identification of Moving Patterns on a Fingertip-based Electro-Tactile Display Array
}

\author{
Mehdi Rahimi, Fang Jiang and Yantao Shen
}

\begin{abstract}
An electro-tactile display can be used to stimulate sensations in the skin. The ultimate achievement in this area is to open a new information communication channel using this sensory substitution system. One of the requirement of such communication channel is to deliver meaningful commands to the user. The sensations should be distinctive enough to be readily understandable for the operator. This study is perusing the feasibility of generating identifiable moving patterns in the electrotactile display. Then, the degree of identification performed by the users will be validated.
\end{abstract}

An electro-tactile display is built using an array of sixteen contacts to form a moving pattern by delivering electrical signal to the fingertip skin. This signal can have varying voltages, frequencies or duty cycles to form the most comfortable sensation. Moving patterns can be generated by individually or collectively toggling the electrical contacts on the electro-tactile display. This will achieve a stimulation of a moving pattern. In this regard, a moving pattern can be compared to a set of frameby-frame pictures that construct a movie. Similarly, by toggling the contacts in a specific order, a moving pattern can be achieved.

In this study, eight subjects participated. A questionnaire was used to assess the sensation of the corresponding movement. The results of these reports were analyzed and a conclusion regarding the identification of the direction of the movement was drawn. It became clear that the direction of the movement had a significant impact on the recognition of the patterns.

Furthermore, an analysis of the detection threshold (DT) voltage and current mapping was performed to evaluate the effect of the internal structure of the skin for each user on the assessment performance. Based on the mapping results, it became clear that the DT voltage is vastly different for each contact and the resulting spatial map is also unique to each user.

\footnotetext{
*This research work was supported by NSF CAREER Award CBET\#1352006 and the National Eye Institute of the National Institute of Health under award R01EY026275.

${ }^{1}$ M. Rahimi and Y. Shen are with the Department of Electrical and Biomedical Engineering, University of Nevada, Reno, Reno, NV, 89557 USA e-mails: mrahimiesamic.org, ytshen@unr. edu

${ }^{2} \mathrm{~F}$. Jiang is with the Department of psychology, University of Nevada, Reno, Reno, NV, 89557 USA e-mail: fang jeunr.edu
}

\section{INTRODUCTION}

$\mathbf{T}$ HE principles of an electro-tactile display have been investigated in several studies [1] and various applications have been suggested based on an electrotactile display [2]. It has been found that the electrotactile approach has many significant advantages over a mechanical display. One of those, is the shorter time that an electrical signal needs to update in comparison to a mechanical signal with many moving parts. The skin would also react to electrical signal significantly faster and with more accuracy [3][4]. Other benefits of the electrical stimulation include higher efficiency, flexibility, and convenience [5]. Still, there are some shortcomings in regards to the constraints of using electrical stimulation. One of those, is the importance of the location of the contacts on the skin [6].

Also, the size and the shape of them is very important [7]. In this regard, some studies chose the approach of either the anode or cathode stimulation where the return electrodes are put in a very close proximity [8]. The main goal in such approaches is to stimulate the nerves in different parts to induce various feelings such as vibration or pressure [9]. This is a viable approach but it will introduce a new challenge as a side effect. Having the return electrodes in close distance to the anode or cathode electrodes will limit the available area for the electro-tactile display. This is because of the size of the fingertip and the two-point discrimination threshold (TPDT) of the skin in that area [10]. In this work, we decided to put the contacts slightly distal to the fingertip vortex. As for the return electrode, a single contact is placed on the palm of the hand. This would give the advantage of providing a larger space on the fingertip to build a bigger display. This practice has been tried in many previous studies [11][12].

Another aspect of research in this area is the shape and pattern identification on an electro-tactile display. This has been an interest to many researchers [13][14]. These displays were designed for various parts of the body such as forehead [15], tongue [16], forearm [17], abdomen [18], and other parts. The tongue, instead of the skin touch, has been selected in several studies because 
the nerves in the tongue use a different pathway for sending the signals to the brainstem [19]. The tongue also requires less voltage to stimulate [20] and the saliva provides a constant conductive environment [21]. Still, using the tongue for electro-tactile displays introduces a high discomfort level for the users. That is one of the reasons that building an electro-tactile display for the fingertip has become an interest to researchers [22]. This study uses the fingertip as the location to stimulate the skin nerves through an electro-tactile display.

Pattern recognition has been investigated in several studies [23] although most of them were concerned with circular movements [24]. But still, a moving pattern identification through an electro-tactile display has not been addressed in these studies. This work investigates how the participants can recognize a moving pattern on an electro-tactile display and whether or not the direction of the movement has any effects on the identification. This will lead to more optimized transfer of commands to the users in the related applications [25]. Various moving patterns with different directions are studied and the analysis of the results shows that the orientation of the movement has a major impact on recognizing the direction.

This paper is structured into the following sections: In Section II, we discuss the methodology of the experiments and systems that are used in this study. Section III presents the results obtained from the analysis and includes a discussion about them. In Section IV we conclude the study.

\section{Methods}

\section{A. Hardware and Systems}

An electro-tactile display with 16 contacts is built to stimulate the fingertip skin and conduct the experiments.

The contacts are fabricated as copper circles with diameters of $1.5 \mathrm{~mm}$. This satisfies the two-point discrimination requirements for the fingertip [10]. A singlepoint return electrode is placed on the palm of the hand. This electrode is a copper circle with a diameter of $3 \mathrm{~cm}$. This display is designed using a printed circuit board (PCB) along with a switching board.

Fig. 1 shows the switching board and the contacts that were used in this study.

One of the functions of the switching board is to amplify the input signal. The input signal is either provided from a counter/decoder IC or a function generator (UNi AFG-1010). This provides a choice between using a function generator or having a stand-alone circuit. A single-board computer, Raspberry Pi 3, connected to a visual display is used to control the switching board and

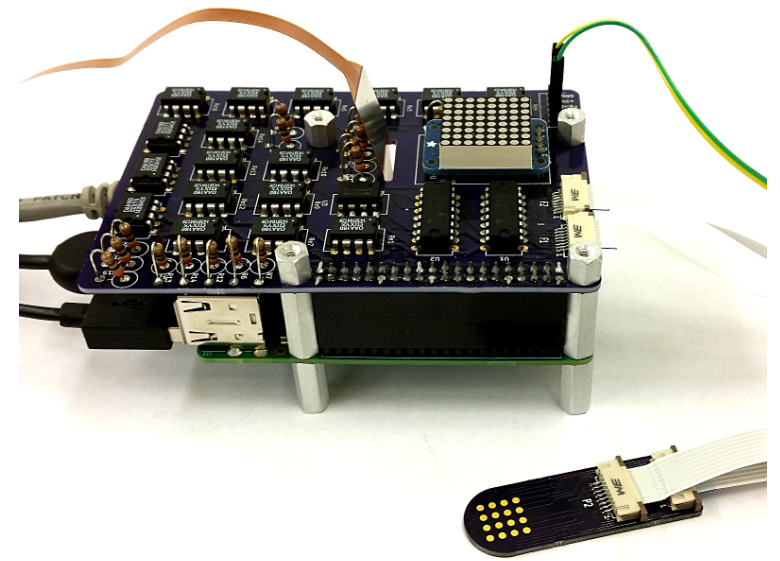

Fig. 1. The contacts are connected to the switching board which is placed on top of a Raspberry Pi. The board also provides a display to give a visual feedback.

also providing a visual feedback. This helps to know which contacts are switched on at any given time.

The 16 contacts on the fingertip piece are designed as a $4 \times 4$ array. A sketch of the system is shown in Fig. 2 .

The principle of work is that the function generator or the counter/decoder switches the relays on and off. The relays are connected to a high voltage DC power supply (TTi PLH250) which can generate any DC voltages ranging from 0 to 250 Volts with an accuracy of $0.1 \mathrm{~V}$. This results in having a pulse signal with a very high peak-to-peak voltage. The current output on the power supply is limited to $0.2 m A$ in order to protect the circuits and the subjects. This acts as a safeguard in case of an accidental surge in power usage. To monitor the amount of the current that goes through the skin, an ammeter (MASTECH MS217) is used. The function generator has an accuracy of $0.0001 \mathrm{~Hz}$ for the frequency and an accuracy of $1 \%$ for the duty cycles.

\section{B. Participants}

In these experiments, 8 subjects $(7$ males and 1 female) participated. They were all healthy graduate students at the University of Nevada, Reno. There were no reports of any physical problems and they neither had any complications nor showed signs of any issues with their skin. Participation was with informed consent and followed protocols approved by the University of Nevada, Reno Institutional Review Board. The goal and the procedure of the experiment were explained to the participants thoroughly. A cardboard was placed between them and the system to prevent them from seeing the displays and the set parameters. 


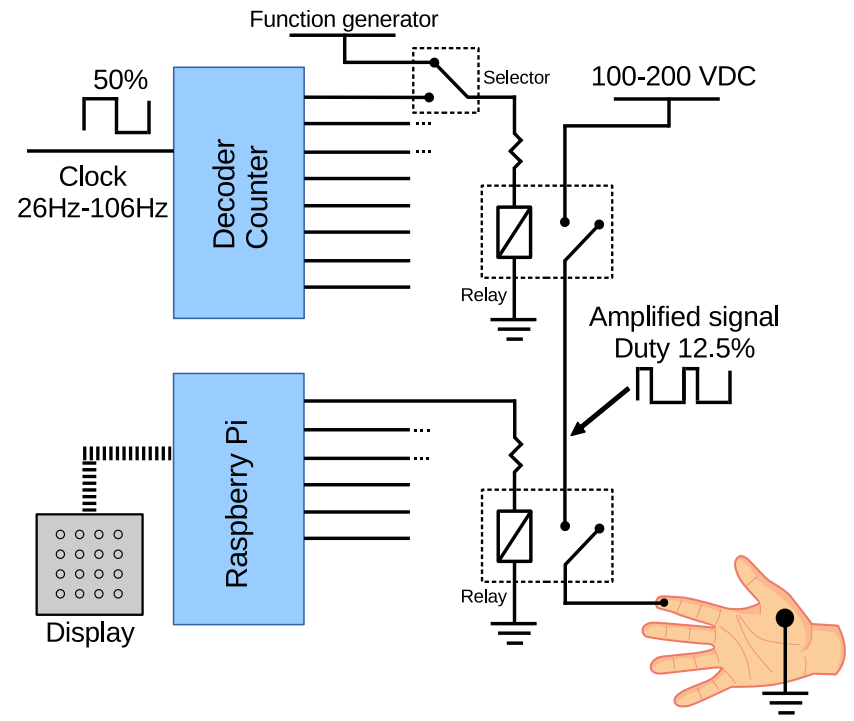

Fig. 2. A sketch of the system is shown here. A choice between the function generator or the counter/encoder can be made. This is to control the distribution of the signal by managing the duty cycle. The Raspberry Pi program commands the relays which connect the signal from the power supply to the fingertip contacts.

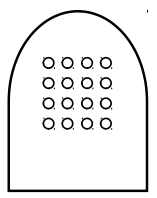

a

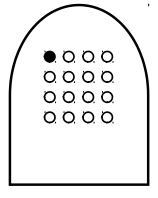

C

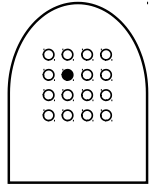

d

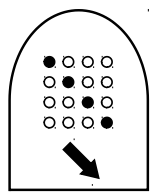

b

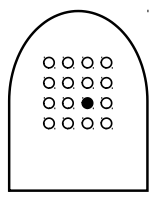

e

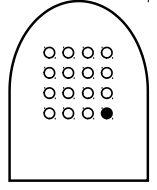

f
Fig. 3. The contacts on the fingertip along with a sample pattern are shown in this sketch. Figures are as follows: a) The default or off mode of the display. b) A sample movement of "Left-Top to BottomRight" is shown. c-f) To generate the movement of the figure (b), these contacts have to turn on and off subsequently with a delay.

\section{Moving Patterns}

By utilizing the $4 \times 4$ contacts on the finger, various patterns were generated and delivered to the finger. This was done by switching the contacts on and off in a specific order to form a pattern. Fig. 3 shows how a movement pattern was generated.

All of the patterns were defined in a program that was run on the Raspberry Pi. The pattern can be easily designed or changed based on need.

There are two time delays that can be defined here. One is the amount of time that a single contact is on. The other delay is the time of switching between

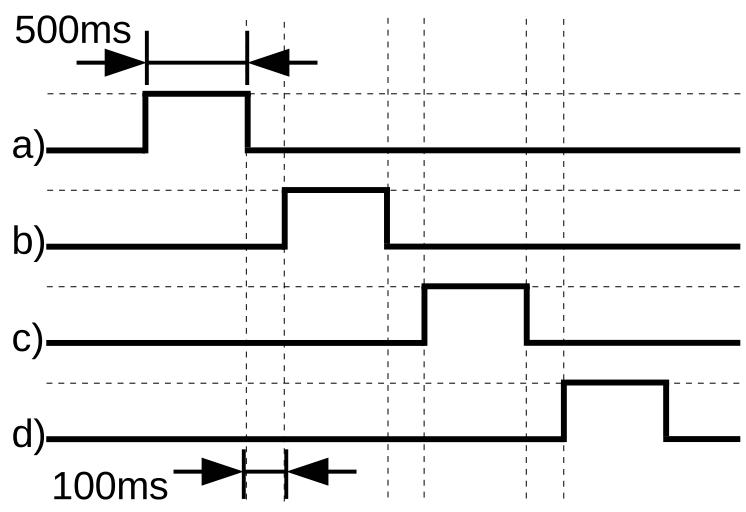

Fig. 4. To generate a moving pattern, four signals are sent to the relays in a sequence as shown in signals (a) to (d). The specified delays of $500 \mathrm{~ms}$ and $100 \mathrm{~ms}$ can be adjusted by the user. Although this is just an example for better illustration, these signals (a to d) can be corresponded with movements (c to f) in Fig. 3 .

two sequencing contacts. These delays were selected as $500 \mathrm{~ms}$ and $100 \mathrm{~ms}$ respectively. This sequence can be seen in Fig. 4.

\section{Signal Properties}

The purpose of selecting a specific electrical signal in this research is to make sure that the participants are comfortable with the signal and can focus on recognizing the moving patterns.

In our previous study [26][27], we determined the characteristics of a comfortable signal. The results can be seen in Fig. 5. This figure leads to a conclusion that voltage, other than any of the other parameters of a signal, would affect the sensation of feeling comfortable. Fig. 6 shows how the voltage level influences the comfort sensation. This assessment was done on a scale of $0-10$ with number 5 being neutral. It can be seen that lower voltages at the detection threshold represent the most comfortable signal.

"V1" was defined as the detection threshold (DT). This was identified by using a staircase method [28]. " $V 2$ " and " $V 3$ " were defined as $V 2=V 1+10 \%(V 1)$ and $V 3=V 1+20 \%(V 1)$.

Since the difference between the first three signals is neglectable, we chose a signal with a frequency of $30 \mathrm{~Hz}$ and a duty cycle of $10 \%$ at the $V 1$ voltage level to match previous studies.

\section{E. Patterns}

To assess the ability of the participants to recognize the moving patterns, six different movement directions were chosen in this study and presented to the participants for identification. These six patterns were categorized into three groups. 


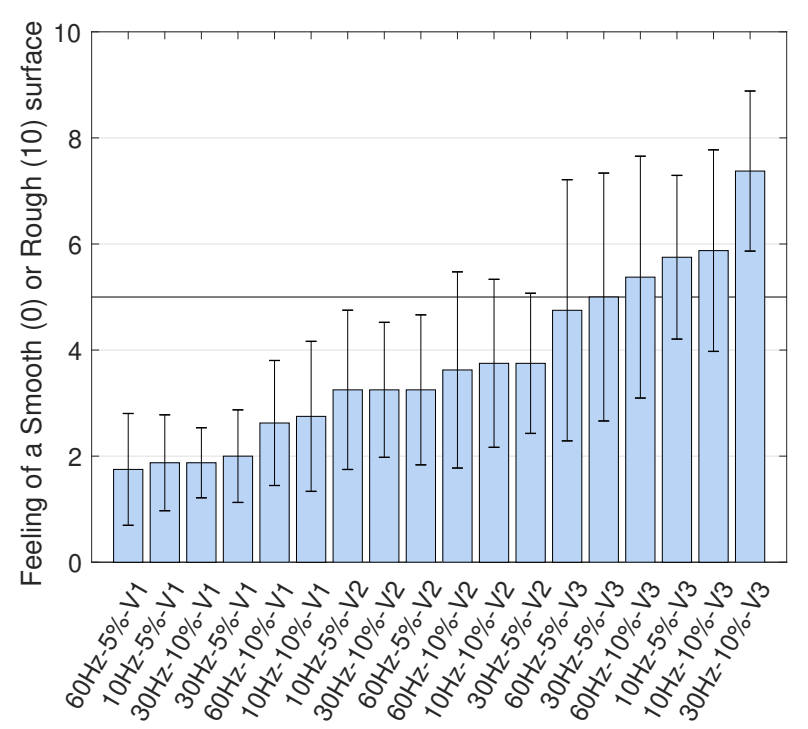

Fig. 5. Various signals were studied to find out which one has the most comfortable sensation for the subject. " $V 1$ " was defined as the detection threshold (DT), the other voltages are $V 2=V 1+10 \%(V 1)$ and $V 3=V 1+20 \%(V 1)$. The assessment was done on a scale of $0-10$ with number 5 being neutral.

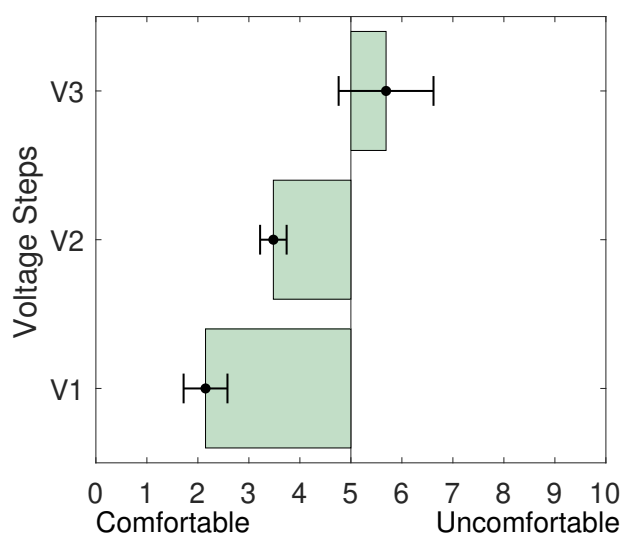

Fig. 6. As we can see in Fig. 5, voltage has the most notable impact on the feeling of comfortable sensation. Here, the effect of the voltage level is shown. The assessment was done on a scale of $0-10$ with number 5 being neutral. It is clear that lower voltage has resulted in a more comfortable sensation $(p<0.001)$.

The first group was the horizontal movements which are orthogonal to the fingertip axis. These include "Right to Left" and "Left to Right" patterns. The second group was the vertical movements (or parallel to the fingertip axis) including "Top to Bottom" and "Bottom to Top". The final and last group was the diagonal movements. This included only two of the four possibilities. The included patterns were "Bottom-Left to Top-Right" and "Top-Left to Bottom-Right".

The inclusion of the diagonal patterns could introduce some challenges for the participants because the
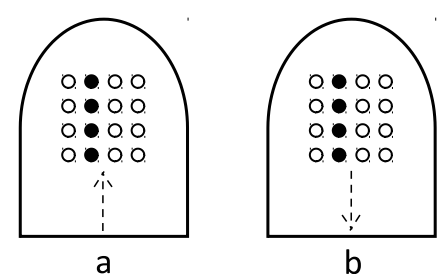

b
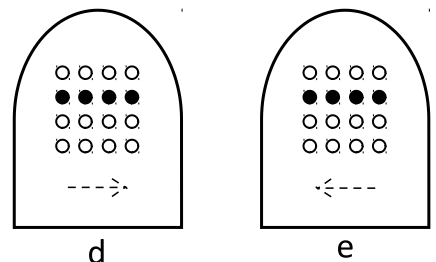

e

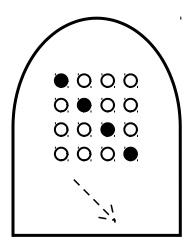

C

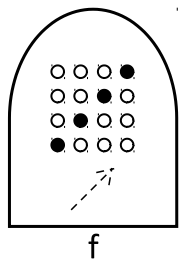

Fig. 7. Six patterns are used in this study. The direction of the dashed arrows shows how the contacts would sequentially switch on and off to generate the pattern. Patterns are: a) Bottom to Top, b) Top to Bottom, c) Top-Left to Bottom-Right, d) Left to Right, e) Right to Left and f) Bottom-Left to Top-Right.

placement of their fingertip could result in perceiving a vertical movement such as a "Bottom to Top" as a diagonal movement such as "Bottom-Left to Top-Right". The $45^{\circ}$ angle might not be enough in some cases if the participant is already placing their finger at an angle to the contacts. To mitigate this problem, extra attention was given to make sure the participants were aware of the problem and placed their finger as much as they can in parallel to the contacts.

The six patterns used in this study are shown in Fig. 7.

\section{F. Questionnaire}

The participants had to fill a short questionnaire after being presented with each signal and recognizing the moving pattern.

The questionnaire had a place for a coded name for the test number along with a place for recording the $V 1$ voltage and the current. Then, the participant could choose the identified pattern from a set of eight figures. This process was repeated for each of the tests. A portion of the questionnaire can be seen in Fig. 8.

It was explained to the participants that they should expect a moving pattern as given in Fig. 3. This was demonstrated to them visually so they could relate that to the electrical sensation.

\section{G. Experimental Procedure}

Each experiment was started by an examination of the system to make sure that all the connections were working properly. Several precautionary practices such 


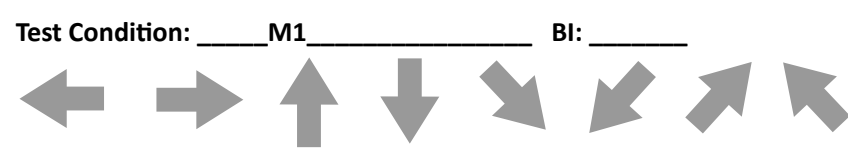

Fig. 8. The questionnaire used in this study is shown here. This was filled by the participants after each and every experiment.

as limiting the current on the power supply and putting a fuse in series to the circuit were in place to make sure of the safety of the participants.

Each subject was briefed thoroughly on how the system works and what he or she is going to experience. Also, the goal of the experiment and the process were discussed. The questionnaire and how the participant was supposed to complete it was also explained to mitigate any misunderstandings.

All of the electro-tactile display contacts and the participants' fingertips and their palms were cleaned using Ethyl Alcohol 200 Proof and were left to dry.

It is noteworthy that the experiments were conducted under cutaneous passive haptic condition. Meaning that the finger was placed directly on the contacts and there was no exploring involved [29]. Still, because of the fact that the contacts were on a flat PCB board, the curvature of the fingertip was not followed.

The experiment was first begun by determining the detection threshold (DT) voltage (i.e V1). Subjects were allowed a short time between 5-20 seconds to examine the feeling of the electrical signal.

The current needed at the DT voltage was recorded for each participant too. This was done to measure the electrical power needed for each individual to reach the threshold point. The results of the measurements of the DT voltage and power is shown in Fig. 9. As it can be seen in that figure, the range of the power needed is very limited for all the participants. The average is $1.954 \mathrm{~mW}$ with a standard deviation of $0.522 \mathrm{~mW}$. On the other hand, the range of the voltage required to reach the DT point is very scattered. The average of the DT voltage is $79.25 \mathrm{~V}$ with a standard deviation of $15.13 \mathrm{~V}$. This resulted in having voltages as low as 52 or as high as 91 volts.

When the participants were ready to begin the experiment, a pattern was given to the contacts randomly and the subjects were allowed to spend a maximum of 30 seconds to analyze the feeling. After filling the questionnaire for that text, another pattern was given to the fingertip.

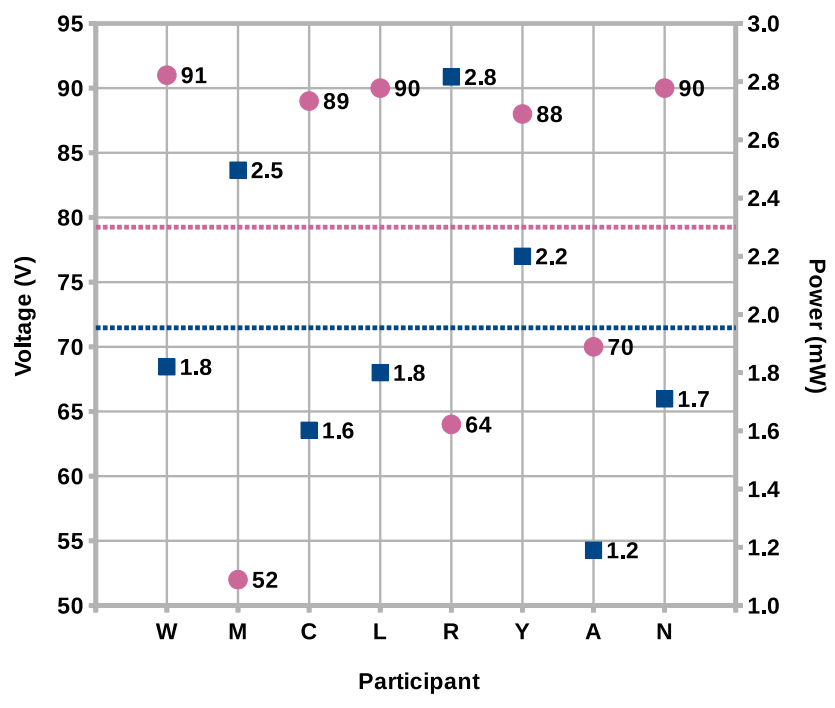

Fig. 9. The detection threshold (DT) voltage and the power used to reach the DT point for each of the participants is shown here. The pink circles show the DT voltages while the blue squares show the power used. The blue dashed line is the average $(1.954 \mathrm{~mW})$ of power for all the subjects. The pink dashed line is the average $(79.25 \mathrm{~V})$ of DT voltages.

\section{RESUltS AND DisCUSSION}

\section{A. Analyzing Directional Movements}

To conduct the analysis, we used a procedure to grade the questionnaires based on the answers provided by participants. Each correct answer was given a score of 1 and each incorrect answer did not receive any scores. The weighted results were accumulated to show the percentage of the correct answers.

Analyzing the results, it quickly became obvious that it is very hard for the participants to understand the angle of the movement rather than the general direction. For example, a movement that is diagonally from BottomLeft to Top-Right can also be understood as simply a Bottom to Top movement. Although care was given to make sure the finger is in parallel to the contacts, but still, the recognition and identification of the direction is shown to be very hard for the participants. To account for this issue, all the results are analyzed in two manners. One is the exact direction as we gave the stimuli and another one is the rough direction for each stimulus by discarding the differences in angles.

Overall, $39.6 \%$ of the participants could identify the exact direction of the movement. Accounting for the rough directions, $60.4 \%$ of the participants were able to identify the direction.

Fig. 10 shows the results of all the moving patterns.

It can be seen that all of the rough answers received higher scores than the exact answers. Also, it is notable 


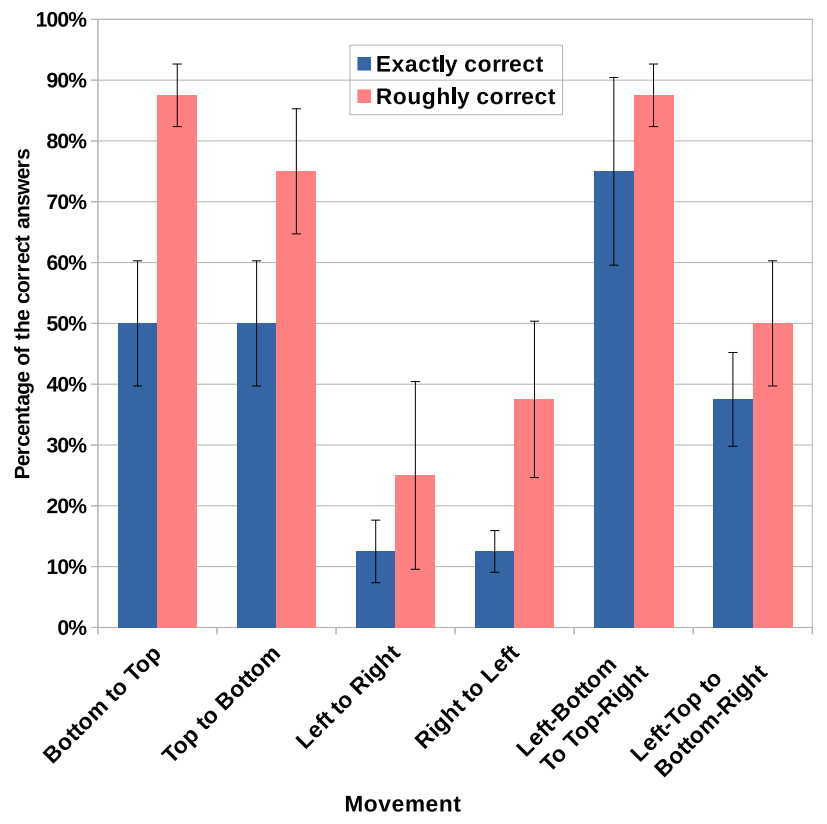

Fig. 10. The comprehensive results of all the tests across all the participants is shown here. The distinction between the "Exact" and "Rough" is explained in the Section III.

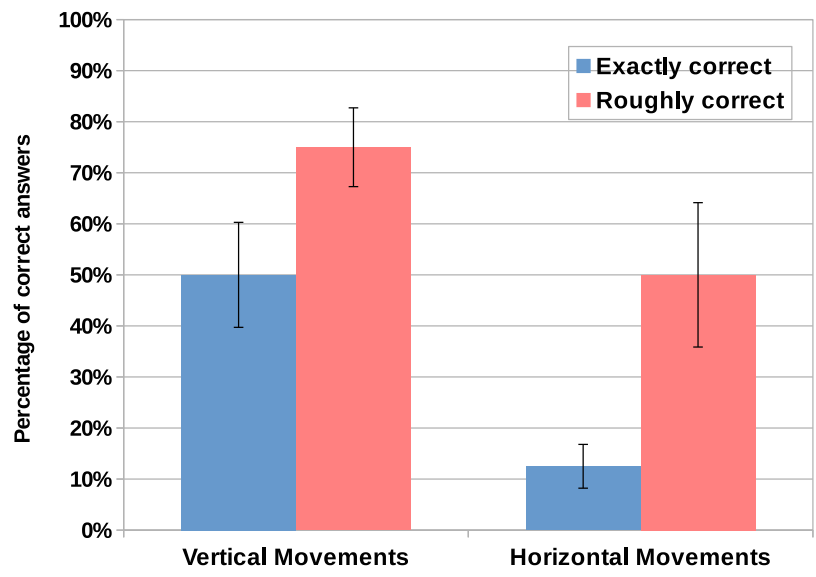

Fig. 11. A summery of the results of answers to the direction of movement is shown here. This is a good illustration that shows the vertical movements were recognized much easier than the horizontal ones.

that some directions had better responses than others.

Considering Fig. 10, we can see that vertical movements could be understood and recognized much easier than the horizontal movements. To better assess this idea, the answers for the general directions were combined to see the effect. This is shown in Fig. 11.

For a vertical movement, $50.0 \%$ of the participants answered exactly right. Combining the direction to form a rough direction for a vertical movement resulted in $75.0 \%$ of correct answers.

On the other hand, for the horizontal movement, only
$12.5 \%$ of the answers were exactly right. The same result for the rough answers in a horizontal movement is $50.0 \%$.

Considering these results, it is obvious that subjects could identify vertical movement better than the horizontal movements.

One reason for this might be the fact that as the person puts his or her fingertip on the contacts, different pressures will be applied to various contacts. Because of the natural curvature of the finger, the contacts that are positioned laterally to the fingertip would not receive homogenous pressure. Meanwhile, the contacts that are placed vertically would get equal pressure.

The amount of the pressure that the contacts receive from the skin is important. That is because the different pressures for each parts means that they get inconsistent currents going through the skin. If all the contacts receive similar high pressure, the current would be also high for all of them and that would affect the detection threshold voltage. But if some of them get lower pressure and some get higher pressure, the distribution of current would not be similar for all of them.

The amount of the current received is noteworthy because it can cause different stimulation.

This inconsistency in the pressure and consequently the dissimilarity in current might be the cause of not recognizing the horizontal movements as good as the vertical movements.

Unless we resort to flexible materials for building the contacts, we will expect the same results due to the natural curvature of the finger.

Considering these results, it is clear that the recognition of the pattern in a vertical alignment to the fingertip is a better approach for pattern recognition.

In this study, we also examined the voltage and power used to stimulate the participants' skins. The electrotactile sensation is based on the current that goes through the skin and since there is an impedance associated with the skin and the flesh, the power consumed can be measured too. The effects of voltage and current on the skin has been investigated by other researchers extensively [30]. Here, we saw that the range of the voltage that was required for different participants to reach the detection threshold is vastly scattered by having a difference of about $40 \mathrm{~V}$. This is an indication of how the different people's skin can grossly differ as a result of multiple factors. These factors can include, but are not limited to, age, sex, amount of sweat, cleanliness of the skin, time of the day, last meal, being hydrated or not and other factors. Considering all these factors in a study such as this, is beyond the scope of this work but some considerations have been taken into account. 
For example, before conducting the experiments, every participants' fingertip and palm were cleaned with alcohol. Also, the range of age of the participants was very narrow considering the fact that they were all college students. Only one of the participants was a female and the rest were males.

The range of DT voltage in our study is very large despite the fact that some precautionary practices were taken. It can be assumed that in given a circumstance if these practices were not followed, the range of DT voltage would be even larger.

\section{B. Voltage and Current Mapping}

Another aspect of an electro-tactile display to consider is the fact that each electrode would require a different DT voltage and takes a different amount of current. This was examined and verified by experiments.

Two procedures were performed in the experiments. One is to determine the detection threshold (DT) voltage for each contact that is in touch with the skin and the other one is to find the amount of current that passes through each contact when a constant voltage is applied.

To determine the detection threshold (DT) voltage, the subject's fingertip was placed on the display (shown in Fig 12) and the voltage was increased gradually. The subjects were asked to express the earliest indication of any sensations. Subjects were allowed a short time between 5 to 20 seconds to examine the feeling of the electrical signal and adjustments were applied. The voltage was then recorded for that contact and the same procedure was repeated for the next contact.

For the second part of the experiments, a same constant voltage was applied to all of the contacts and the current passing through each one was recorded. The constant voltage is set as a low value, considerably below the DT voltage, so that the participants will not feel any sensations.

There are several precautionary practices in place such as limiting the current on the power supply and using a fuse in series to the circuit to make sure of the safety of the participants.

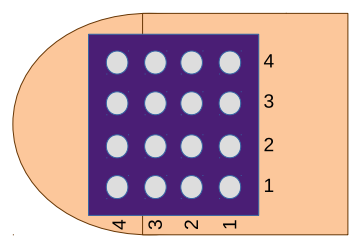

Fig. 12. The electro-tactile display used in these experiments is shown here. The position numbers are corresponding to the voltage and current figures to follow.
1) DT Voltage Mapping: Multiple experiments were run to determine the detection threshold (DT) voltage for each contact and for each participant. It became instantly clear that the DT voltage is not the same for each contact. An example of such an experiment is shown in Fig. 13.
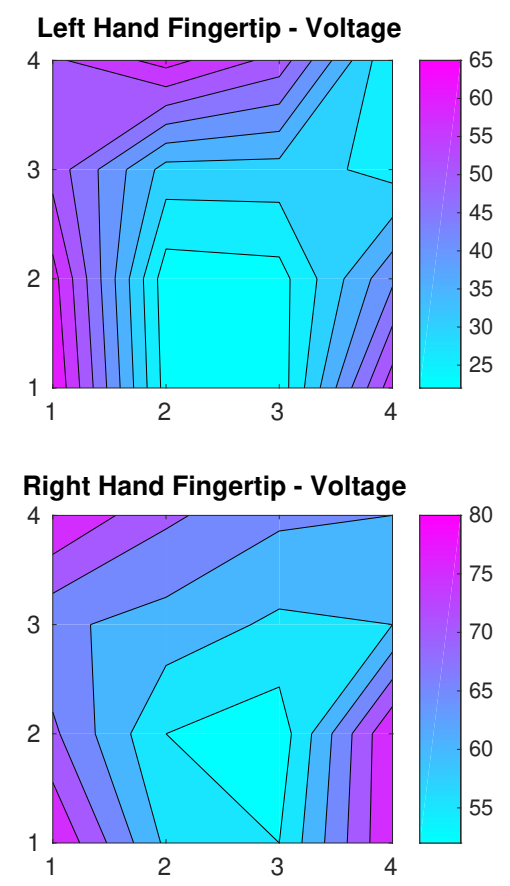

Fig. 13. An example of the DT voltage for subject (a) is shown here. The range for the left hand fingertips (index finger) is from 22 to 65 Volts and the range for the right hand fingertips is from 52 to 80 Volts. The $\mathrm{X}$ and $\mathrm{Y}$ axis represent the position of the contacts.

Noting this figure, it is clear that the voltage for each part of the display is different. There is a 30 to 40 volts difference between different parts of the display. In this specific instance, the voltage that was needed for the subject to feel any sensations was smaller in the center of the fingertip for both hands and larger for the surrounding contacts.

One noteworthy observation in this instance is that the DT voltage map for the right hand and left hand for this participant is not the same, although they may seem similar. This finding was also seen in other participants.

The differences in the DT voltage for each contact can be explained by considering the fact that the tissue of the skin is not the same at every point of the fingertip. The sweat glands and the fat under the skin can play significant roles for increasing or decreasing the impedance of the whole skin at any given point. Another factor to consider is the inequality of the pressure that is applied to the skin tissue. One participant may have a habit of putting pressure on the center of the fingertip or one of the sides. This can change the intensity of the inner tissues and therefore effect the impedance accordingly. 
One other factor can be the distribution of the nerve cells in the skin. Since the DT voltage is dependent on the sensation that the participant receives from the voltage, the distribution of the nerve cells under the skin and their distance to the each contact can play a role too.

There may be many other factors that are involved in this phenomena which are beyond the scope of this paper but they can be investigated more in future research.

The test of finding the DT voltage map was done on both hands of each participant and similar results were obtained as shown in Fig. 13.

Fig. 14 shows this mapping for two of the other participants, both on the right hand. Since each of these maps are unique, only two of them are shown here to emphasize the difference between them.
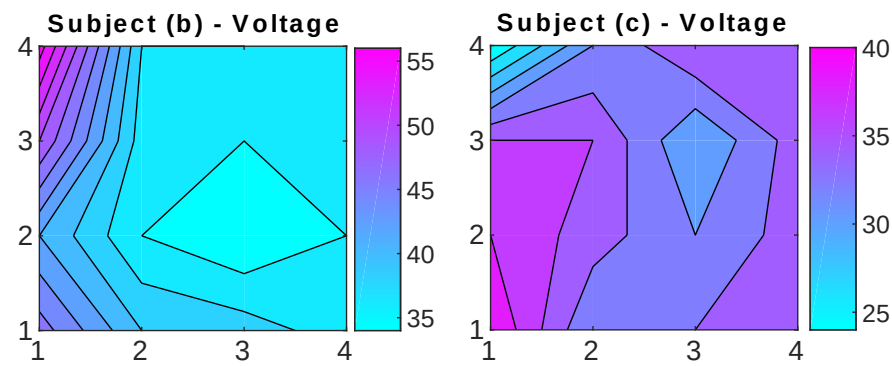

Fig. 14. The DT voltage mapping was performed on all of the participants. Here, two of these maps (for the right hand) are presented as an example to show that each map is unique in its shape and voltage range.

As it can be seen in Fig. 14, the DT voltage map is thoroughly different and unique for each participant. The distribution and also the voltage range are vastly dissimilar. The same phenomena was observed for each of the other participants.

Considering Fig. 13 and Fig. 14, leads to this realization that using a constant voltage for all of the contacts is not an option and to achieve a same sensation for every contact, a specific voltage needs to be applied.

2) Current Mapping: Another approach for achieving a spatial mapping of the fingertip is to use a same constant voltage for all of the contacts and measuring the current that goes through each of them.

In this case, a voltage was selected below the DT voltage level so that the participants could not feel any sensations.

Based on the previous finding, the voltage range for each part of the fingertip can be significantly different from the other parts and using a high voltage or the DT voltage itself, that is comfortable for one part of the skin, can cause uncomfortable sensations in some other parts of the skin.

Based on the conducted tests, it was determined that the comfortable tolerance range for the current on one contact is roughly $10 \mu \mathrm{A}$. This means that provided that the user is receiving a comfortable sensation at $20 \mu \mathrm{A}$, the current can then have a fluctuation ranging from $15 \mu \mathrm{A}$ to $25 \mu \mathrm{A}$. Any currents less than this range will not provoke any sensations for the user and any currents higher than the range will cause an uncomfortable sensation.

Since the acceptable range for the current is very narrow, a highly accurate, fast, and efficient mean for measuring the current is needed to make sure the user is not receiving any currents out of that specific range.

To achieve this, a differential ADC based on the MCP3424 microchip was used. The current passes a shunt resistor and the voltage drop across is sampled, amplified, and passed to the ADC. The output is read by the program on the Raspberry Pi.

Fig. 15 shows the resulting maps of the measurements of the current. Once again, it can be seen that none of these maps are similar whether in regard to the shape and distribution or the range of the minimum and maximum current values.

Another noteworthy observation is that since the current mapping was not conducted at the same voltage as the DT voltage map, the corresponding figures do not necessarily match. This can be explained by considering the fact that higher voltages have a more pronounced impact on the tissue, whereas lower voltages may not effect the tissue as significantly. This difference can lead to different maps as for the current or voltage.

\section{CONCLUSION}

This work examined the identification and recognition of various moving patterns induced by electrical signals. These signals were delivered to the fingertip skin through an electro-tactile display. The signal properties were selected according to previous studies. The hardware and software required for this experiment were designed and implemented in the lab.

An experiment with the participation of 8 subjects was conducted. The subjects received the electrical signals on their fingertips and were asked to fill a questionnaire to assess the direction of the moving patterns. Analyzing the results of the questionnaire showed that the direction of the pattern is a governing factor in the recognition and identification of the moving pattern.

It became clear that subjects had more difficulty in identifying the movement of the pattern if the direction was moving horizontally rather than vertically. This was further verified by combining the results of the movements by disregarding the effects of the diagonal patterns.

It was reasoned that the challenge of identifying horizontal movement is potentially due to the inconsistency 

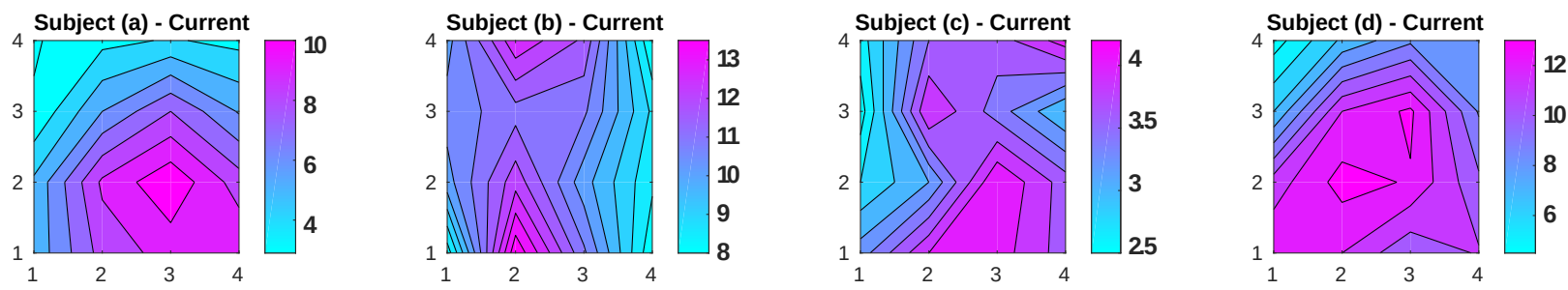

Fig. 15. A same constant voltage was used to measure the current that goes through each contact and generating the resultant map. The same was done for all of the participants and the result is shown here. As it can be seen, none of the participant had a similar pattern in this regard. All currents are in $\mu A$.

of the pressure that the contacts receive as a result of the natural curvature of the fingertip. A solution for this particular situation might be using a flexible material to build the electro-tactile display. This would mitigate the inconsistency of the pressure applied to the contacts as long as the fingertip is not placed on a hard surface such as a table.

Also, experiments were designed to measure the current that goes through each contact of the electrotactile display. Through these experiments the detection threshold (DT) voltage for each contact was determined for every participant. Based on those results, it became clear that the DT voltage is vastly different for each contact and the resulting spatial map is also unique to each user.

To conclude, we addressed the differences in identification of the moving patterns generated by electro-tactile stimulation. Our results suggest that the direction of a moving pattern is an important factor for its recognition.

\section{ACKNOWLEDGMENT}

The authors would like to thank the participants in this study. The participation was with informed consent and followed protocols approved by the University of Nevada, Reno Institutional Review Board.

\section{CONFLICT OF INTEREST STATEMENT}

The authors declare that the research was conducted in the absence of any commercial or financial relationships that could be construed as a potential conflict of interest.

\section{REFERENCES}

[1] P. Bach-y Rita, M. E. Tyler, and K. A. Kaczmarek, "Seeing with the brain," International journal of human-computer interaction, vol. 15, no. 2, pp. 285-295, 2003.

[2] H. Kajimoto, N. Kawakami, T. Maeda, and S. Tachi, "Electrotactile display with tactile primary color approach," Graduate School of Information and Technology, The University of Tokyo, 2004.

[3] A. Y. Szeto and F. A. Saunders, "Electrocutaneous stimulation for sensory communication in rehabilitation engineering," IEEE Transactions on Biomedical Engineering, no. 4, pp. 300-308, 1982.
[4] K. A. Kaczmarek, J. G. Webster, P. Bach-y Rita, and W. J. Tompkins, "Electrotactile and vibrotactile displays for sensory substitution systems," IEEE Transactions on Biomedical Engineering, vol. 38, no. 1, pp. 1-16, 1991.

[5] K. A. Kaczmarek, "Sensory augmentation and substitution," CRC handbook of biomedical engineering, pp. 2100-2109, 1995.

[6] A. Y. Szeto and R. R. Riso, "Sensory feedback using electrical stimulation," Rehabilitation engineering, p. 29, 1990.

[7] C. Collins and F. Saunders, "Pictorial display by direct electrical stimulation of the skin," J. Biomed. Syst, vol. 1, no. 2, pp. 3-16, 1970.

[8] H. Kajimoto, N. Kawakami, and S. Tachi, "Psychophysical evaluation of receptor selectivity in electro-tactile display," in Proc. of 13th International Symposium on Measurement and Control in Robotics (ISMCR), vol. 13, 2003, pp. 83-86.

[9] H. Kajimoto, N. Kawakami, T. Maeda, and S. Tachi, "Electrotactile display with force feedback," in Proc. World Multiconference on Systemics, Cybernetics and Informatics (SCI2001), vol. 11, 2001, pp. 95-99.

[10] M. Solomonow, J. Lyman, and A. Freedy, "Electrotactile twopoint discrimination as a function of frequency, body site, laterality, and stimulation codes," Annals of biomedical engineering, vol. 5, no. 1, pp. 47-60, 1977.

[11] H. Kajimoto, N. Kawakami, T. Maeda, and S. Tachi, "Electrocutaneous display with receptor selective stimulations," Electronics and Communications in Japan (Part II: Electronics), vol. 85, no. 6, pp. 40-49, 2002.

[12] K. B. Shimoga, "A survey of perceptual feedback issues in dexterous telemanipulation. ii. finger touch feedback," in Virtual Reality Annual International Symposium, 1993., 1993 IEEE. IEEE, 1993, pp. 271-279.

[13] H. Takahashi, H. Kajimoto, N. Kawakami, and S. Tachi, "Electro-tactile display with localized high-speed switching," in Proc. of ICAT, 2002, pp. 10-16.

[14] K. A. Kaczmarek and S. J. Haase, "Pattern identification and perceived stimulus quality as a function of stimulation waveform on a fingertip-scanned electrotactile display," IEEE Transactions on Neural Systems and Rehabilitation Engineering, vol. 11, no. 1, pp. 9-16, 2003.

[15] H. Kajimoto, Y. Kanno, and S. Tachi, "Forehead electro-tactile display for vision substitution," in Proc. EuroHaptics, 2006.

[16] P. Bach-y Rita, K. A. Kaczmarek, M. E. Tyler, and J. GarciaLara, "Form perception with a 49-point electrotactile stimulus array on the tongue: a technical note," Journal of rehabilitation research and development, vol. 35, no. 4, p. 427, 1998.

[17] G. Ng, P. Barralon, G. Dumont, S. K. Schwarz, and J. M. Ansermino, "Optimizing the tactile display of physiological information: vibro-tactile vs. electro-tactile stimulation, and forearm or wrist location," in Engineering in Medicine and 
Biology Society, 2007. EMBS 2007. 29th Annual International Conference of the IEEE. IEEE, 2007, pp. 4202-4205.

[18] S. J. Haase and K. A. Kaczmarek, "Electrotactile perception of scatterplots on the fingertips and abdomen," Medical and Biological Engineering and Computing, vol. 43, no. 2, pp. 283289, 2005.

[19] R. Velázquez, "Wearable assistive devices for the blind," Wearable and autonomous biomedical devices and systems for smart environment, vol. 75, pp. 331-349, 2010.

[20] E. Sampaio, S. Maris, and P. Bach-y Rita, "Brain plasticity:visualacuity of blind persons via the tongue," Brain research, vol. 908, no. 2, pp. 204-207, 2001.

[21] K. Kaczmarek, "The tongue display unit (tdu) for electrotactile spatiotemporal pattern presentation," Scientia Iranica, vol. 18, no. 6 , pp. 1476-1485, 2011.

[22] H. Kajimoto, N. Kawakami, S. Tachi, and M. Inami, "Smarttouch: Electric skin to touch the untouchable," IEEE computer graphics and applications, vol. 24, no. 1, pp. 36-43, 2004.

[23] K. A. Kaczmarek, M. E. Tyler, and P. Bach-y Rita, "Pattern identification on a fingertip-scanned electrotactile display," in Engineering in Medicine and Biology Society, 1997. Proceedings of the 19th Annual International Conference of the IEEE, vol. 4. IEEE, 1997, pp. 1694-1696.

[24] M. A. Schweisfurth, R. Schweizer, and S. Treue, "Feature-based attentional modulation of orientation perception in somatosensation," Frontiers in human neuroscience, vol. 8, 2014.

[25] M. Rahimi, Z. Liu, F. Jiang, and Y. Shen, "Finger-eye: Design, implementation and evaluation of an electro-tactile system enabling the blind and visually impaired to read printed text," Manuscript submitted for publication, 2019.

[26] M. Rahimi, F. Jiang, and Y. Shen, "Analysing the perceptual attributes of electro-tactile stimuli as function of various signal properties," in submitted to IEEE Transactions on Neural Systems and Rehabilitation Engineering, 2019.

[27] — "Adaptive spatial mapping of electro-tactile threshold based on subdivision bio-impedance feedback," in Real-time Computing and Robotics (RCAR), IEEE International Conference on. IEEE, 2019.

[28] T. N. Cornsweet, "The staircase-method in psychophysics," The
American journal of psychology, vol. 75, no. 3, pp. 485-491, 1962.

[29] M. Symmons, "Active and passive haptic exploration of two-and three-dimensional stimuli," Ph.D. dissertation, Monash University, 2004.

[30] Y. A. Chizmadzhev, A. V. Indenbom, P. I. Kuzmin, S. V. Galichenko, J. C. Weaver, and R. O. Potts, "Electrical properties of skin at moderate voltages: contribution of appendageal macropores," Biophysical Journal, vol. 74, no. 2, pp. 843-856, 1998.

Mehdi Rahimi is a $\mathrm{PhD}$ candidate in the Department of Electrical and Biomedical Engineering at the University of Nevada, Reno. He has been a research assistant for the last few years. He has mainly worked on the electro-tactile displays. His research is on analyzing the perceptions associated with the electro-tactile display as well as studying various applications of such systems in the biomedical field.

Fang Jiang is an assistant professor in the Department of Psychology at the University of Nevada, Reno. She joined the faculty in 2015. Her research examines relationship between brain structure and function/behaviors and the mechanisms underlying such relationship, with a particular emphasis on functional relevance of cross-modal responses consequent on sensory deprivation. She uses research methods including neuroimaging and behavioral measures.

Yantao Shen is an associate professor in the Department of Electrical and Biomedical Engineering at the University of Nevada, Reno. Dr. Shen's research is in the areas of Bioinstrumentation \& Automation, Biomechatronics/-robotics, Sensors and Actuators, and Tactile/Haptic Interfaces. His research has been supported by federal agencies such as the National Science Foundation (NSF), National Aeronautics and Space Administration (NASA), as well as the state's and local agencies. Dr. Shen has published more than 90 research papers in the fields. Several of these publications have been nominated for or have won the Best Paper Awards. In addition, Dr. Shen is a recipient of NSF CAREER Award. 